\title{
Small Inductive Dimension of Topological Spaces
}

\author{
Karol Pąk \\ Institute of Computer Science \\ University of Białystok \\ Poland
}

\begin{abstract}
Summary. We present the concept and basic properties of the MengerUrysohn small inductive dimension of topological spaces according to the books [7]. Namely, the paper includes the formalization of main theorems from Sections 1.1 and 1.2 .
\end{abstract}

MML identifier: TOPDIM_1, version: $\underline{7.11 .04 \quad 4.130 .1076}$

The terminology and notation used here are introduced in the following articles: [17], [8], [15], [5], [16], [6], [18], [14], [1], [2], [3], [13], [11], [9], [12], [19], [20], [10], and [4].

\section{Preliminaries}

For simplicity, we adopt the following rules: $T, T_{1}, T_{2}$ denote topological spaces, $A, B$ denote subsets of $T, F$ denotes a subset of $T\left\lceil A, G, G_{1}, G_{2}\right.$ denote families of subsets of $T, U, W$ denote open subsets of $T\lceil A, p$ denotes a point of $T\lceil A, n$ denotes a natural number, and $I$ denotes an integer.

One can prove the following propositions:

(1) $\operatorname{Fr}(B \cap A) \subseteq \operatorname{Fr} B \cap A$.

(2) $T$ is a $T_{4}$ space if and only if for all closed subsets $A, B$ of $T$ such that $A$ misses $B$ there exist open subsets $U, W$ of $T$ such that $A \subseteq U$ and $B \subseteq W$ and $\bar{U}$ misses $\bar{W}$.

Let us consider $T$. The sequence of ind of $T$ yields a sequence of subsets of $2^{\text {the carrier of } T}$ and is defined by the conditions (Def. 1 ). 
(Def. 1)(i) (The sequence of ind of $T)(0)=\left\{\emptyset_{T}\right\}$, and

(ii) $\quad A \in$ (the sequence of ind of $T)(n+1)$ iff $A \in$ (the sequence of ind of $T)(n)$ or for all $p, U$ such that $p \in U$ there exists $W$ such that $p \in W$ and $W \subseteq U$ and Fr $W \in($ the sequence of ind of $T)(n)$.

Let us consider $T$. Note that the sequence of ind of $T$ is ascending.

We now state the proposition

(3) For every $F$ such that $F=B$ holds $F \in($ the sequence of ind of $T\lceil A)(n)$ iff $B \in($ the sequence of ind of $T)(n)$.

Let us consider $T, A$. We say that $A$ has finite small inductive dimension if and only if:

(Def. 2) There exists $n$ such that $A \in($ the sequence of ind of $T)(n)$.

Let us consider $T, A$. We introduce $A$ is finite-ind as a synonym of $A$ has finite small inductive dimension.

Let us consider $T, G$. We say that $G$ has finite small inductive dimension if and only if:

(Def. 3) There exists $n$ such that $G \subseteq$ (the sequence of ind of $T)(n)$.

Let us consider $T, G$. We introduce $G$ is finite-ind as a synonym of $G$ has finite small inductive dimension.

The following proposition is true

(4) If $A \in G$ and $G$ is finite-ind, then $A$ is finite-ind.

Let us consider $T$. One can check the following observations:

* every subset of $T$ which is finite is also finite-ind,

* there exists a subset of $T$ which is finite-ind,

* every family of subsets of $T$ which is empty is also finite-ind, and

* there exists a family of subsets of $T$ which is non empty and finite-ind.

Let $T$ be a non empty topological space. One can check that there exists a subset of $T$ which is non empty and finite-ind.

Let us consider $T$. We say that $T$ has finite small inductive dimension if and only if:

(Def. 4) $\Omega_{T}$ has finite small inductive dimension.

Let us consider $T$. We introduce $T$ is finite-ind as a synonym of $T$ has finite small inductive dimension.

One can verify that every topological space which is empty is also finite-ind.

Let $X$ be a set. Note that $\{X\}_{\text {top }}$ is finite-ind.

One can check that there exists a topological space which is non empty and finite-ind.

In the sequel $A_{1}$ is a finite-ind subset of $T$ and $T_{3}$ is a finite-ind topological space. 


\section{Small Inductive Dimension}

Let us consider $T$ and let us consider $A$. Let us assume that $A$ is finite-ind. The functor ind $A$ yields an integer and is defined as follows:

(Def. 5) $A \in$ (the sequence of ind of $T$ )(ind $A+1$ ) and $A \notin$ (the sequence of ind of $T)($ ind $A)$.

We now state two propositions:

(5) $\quad-1 \leq$ ind $A_{1}$.

(6) ind $A_{1}=-1$ iff $A_{1}$ is empty.

Let $T$ be a non empty topological space and let $A$ be a non empty finite-ind subset of $T$. Observe that ind $A$ is natural.

The following three propositions are true:

(7) ind $A_{1} \leq n-1$ iff $A_{1} \in($ the sequence of ind of $T)(n)$.

(8) For every finite subset $A$ of $T$ holds ind $A<\overline{\bar{A}}$.

(9) ind $A_{1} \leq n$ if and only if for every point $p$ of $T\left\lceil A_{1}\right.$ and for every open subset $U$ of $T\left\lceil A_{1}\right.$ such that $p \in U$ there exists an open subset $W$ of $T\left\lceil A_{1}\right.$ such that $p \in W$ and $W \subseteq U$ and $\operatorname{Fr} W$ is finite-ind and ind $\operatorname{Fr} W \leq n-1$.

Let us consider $T$ and let us consider $G$. Let us assume that $G$ is finite-ind. The functor ind $G$ yielding an integer is defined by the conditions (Def. 6).

(Def. 6$)(\mathrm{i}) \quad G \subseteq($ the sequence of ind of $T$ ) (ind $G+1$ ),

(ii) $-1 \leq \operatorname{ind} G$, and

(iii) for every integer $i$ such that $-1 \leq i$ and $G \subseteq$ (the sequence of ind of $T)(i+1)$ holds ind $G \leq i$.

The following propositions are true:

(10) ind $G=-1$ and $G$ is finite-ind iff $G \subseteq\left\{\emptyset_{T}\right\}$.

(11) $G$ is finite-ind and ind $G \leq I$ iff $-1 \leq I$ and for every $A$ such that $A \in G$ holds $A$ is finite-ind and ind $A \leq I$.

(12) If $G_{1}$ is finite-ind and $G_{2} \subseteq G_{1}$, then $G_{2}$ is finite-ind and ind $G_{2} \leq$ ind $G_{1}$.

Let us consider $T$ and let $G_{1}, G_{2}$ be finite-ind families of subsets of $T$. Observe that $G_{1} \cup G_{2}$ is finite-ind.

The following proposition is true

(13) If $G$ is finite-ind and $G_{1}$ is finite-ind and ind $G \leq I$ and ind $G_{1} \leq I$, then $\operatorname{ind}\left(G \cup G_{1}\right) \leq I$.

Let us consider $T$. The functor ind $T$ yields an integer and is defined as follows:

(Def. 7) $\operatorname{ind} T=\operatorname{ind}\left(\Omega_{T}\right)$.

Let $T$ be a non empty finite-ind topological space. One can verify that ind $T$ is natural.

The following three propositions are true: 
(14) For every non empty set $X$ holds ind $\left(\{X\}_{\text {top }}\right)=0$.

(15) Given $n$ such that let $p$ be a point of $T$ and $U$ be an open subset of $T$. Suppose $p \in U$. Then there exists an open subset $W$ of $T$ such that $p \in W$ and $W \subseteq U$ and $\operatorname{Fr} W$ is finite-ind and ind $\operatorname{Fr} W \leq n-1$. Then $T$ is finite-ind.

(16) ind $T_{3} \leq n$ if and only if for every point $p$ of $T_{3}$ and for every open subset $U$ of $T_{3}$ such that $p \in U$ there exists an open subset $W$ of $T_{3}$ such that $p \in W$ and $W \subseteq U$ and $\operatorname{Fr} W$ is finite-ind and ind $\operatorname{Fr} W \leq n-1$.

\section{Monotonicity of the Small Inductive Dimension}

Let us consider $T_{3}$. Observe that every subset of $T_{3}$ is finite-ind.

Let us consider $T, A_{1}$. Note that $T\left\lceil A_{1}\right.$ is finite-ind.

One can prove the following propositions:

(17) $\operatorname{ind}\left(T\left\lceil A_{1}\right)=\right.$ ind $A_{1}$.

(18) If $T\lceil A$ is finite-ind, then $A$ is finite-ind.

(19) If $A \subseteq A_{1}$, then $A$ is finite-ind and ind $A \leq \operatorname{ind} A_{1}$.

(20) For every subset $A$ of $T_{3}$ holds ind $A \leq \operatorname{ind} T_{3}$.

(21) If $F=B$ and $B$ is finite-ind, then $F$ is finite-ind and ind $F=\operatorname{ind} B$.

(22) If $F=B$ and $F$ is finite-ind, then $B$ is finite-ind and ind $F=\operatorname{ind} B$.

(23) Let $T$ be a non empty topological space. Suppose $T$ is a $T_{3}$ space. Then $T$ is finite-ind and ind $T \leq n$ if and only if for every closed subset $A$ of $T$ and for every point $p$ of $T$ such that $p \notin A$ there exists a subset $L$ of $T$ such that $L$ separates $\{p\}, A$ and $L$ is finite-ind and ind $L \leq n-1$.

(24) If $T_{1}$ and $T_{2}$ are homeomorphic, then $T_{1}$ is finite-ind iff $T_{2}$ is finite-ind.

(25) If $T_{1}$ and $T_{2}$ are homeomorphic and $T_{1}$ is finite-ind, then ind $T_{1}=\operatorname{ind} T_{2}$.

(26) Let $A_{2}$ be a subset of $T_{1}$ and $A_{3}$ be a subset of $T_{2}$. Suppose $A_{2}$ and $A_{3}$ are homeomorphic. Then $A_{2}$ is finite-ind if and only if $A_{3}$ is finite-ind.

(27) Let $A_{2}$ be a subset of $T_{1}$ and $A_{3}$ be a subset of $T_{2}$. If $A_{2}$ and $A_{3}$ are homeomorphic and $A_{2}$ is finite-ind, then ind $A_{2}=$ ind $A_{3}$.

(28) If $T_{1} \times T_{2}$ is finite-ind, then $T_{2} \times T_{1}$ is finite-ind and $\operatorname{ind}\left(T_{1} \times T_{2}\right)=\operatorname{ind}\left(T_{2} \times\right.$ $\left.T_{1}\right)$.

(29) For every family $G_{3}$ of subsets of $T\left\lceil A\right.$ such that $G_{3}$ is finite-ind and $G_{3}=G$ holds $G$ is finite-ind and ind $G=\operatorname{ind} G_{3}$.

(30) For every family $G_{3}$ of subsets of $T\lceil A$ such that $G$ is finite-ind and $G_{3}=G$ holds $G_{3}$ is finite-ind and ind $G=\operatorname{ind} G_{3}$. 


\section{Basic Properties 0-Dimensional Topological Spaces}

Next we state several propositions:

(31) $T$ is finite-ind and ind $T \leq n$ if and only if there exists a basis $B_{1}$ of $T$ such that for every $A$ such that $A \in B_{1}$ holds $\operatorname{Fr} A$ is finite-ind and ind Fr $A \leq n-1$.

(32) Let given $T$. Suppose that

(i) $T$ is a $T_{1}$ space, and

(ii) for all closed subsets $A, B$ of $T$ such that $A$ misses $B$ there exist closed subsets $A^{\prime}, B^{\prime}$ of $T$ such that $A^{\prime}$ misses $B^{\prime}$ and $A^{\prime} \cup B^{\prime}=\Omega_{T}$ and $A \subseteq A^{\prime}$ and $B \subseteq B^{\prime}$.

Then $T$ is finite-ind and ind $T \leq 0$.

(33) Let $X$ be a set and $f$ be a sequence of subsets of $X$. Then there exists a sequence $g$ of subsets of $X$ such that

(i) $\bigcup \operatorname{rng} f=\bigcup \operatorname{rng} g$,

(ii) for all natural numbers $i, j$ such that $i \neq j$ holds $g(i)$ misses $g(j)$, and

(iii) for every $n$ there exists a finite family $f_{1}$ of subsets of $X$ such that $f_{1}=\{f(i) ; i$ ranges over elements of $\mathbb{N}: i<n\}$ and $g(n)=f(n) \backslash \bigcup f_{1}$.

(34) Let given $T$. Suppose $T$ is finite-ind and ind $T \leq 0$ and $T$ is Lindelöf. Let $A, B$ be closed subsets of $T$. Suppose $A$ misses $B$. Then there exist closed subsets $A^{\prime}, B^{\prime}$ of $T$ such that $A^{\prime}$ misses $B^{\prime}$ and $A^{\prime} \cup B^{\prime}=\Omega_{T}$ and $A \subseteq A^{\prime}$ and $B \subseteq B^{\prime}$.

(35) Let given $T$. Suppose $T$ is a $T_{1}$ space and Lindelöf. Then $T$ is finite-ind and ind $T \leq 0$ if and only if for all closed subsets $A, B$ of $T$ such that $A$ misses $B$ holds $\emptyset_{T}$ separates $A, B$.

(36) Let given $T$. Suppose that

(i) $\quad T$ is a $T_{4}$ space, a $T_{1}$ space, and Lindelöf, and

(ii) there exists a family $F$ of subsets of $T$ such that $F$ is closed, a cover of $T$, countable, and finite-ind and ind $F \leq 0$.

Then $T$ is finite-ind and ind $T \leq 0$.

In the sequel $T_{4}$ is a metrizable topological space.

We now state four propositions:

(37) Let $A, B$ be closed subsets of $T_{4}$. Suppose $A$ misses $B$. Let $N_{1}$ be a finiteind subset of $T_{4}$. Suppose ind $N_{1} \leq 0$ and $T_{4} \uparrow N_{1}$ is second-countable. Then there exists a subset $L$ of $T_{4}$ such that $L$ separates $A, B$ and $L$ misses $N_{1}$.

(38) Let $N_{1}$ be a subset of $T_{4}$. Suppose $T_{4}\left\lceil N_{1}\right.$ is second-countable. Then $N_{1}$ is finite-ind and ind $N_{1} \leq 0$ if and only if for every point $p$ of $T_{4}$ and for every open subset $U$ of $T_{4}$ such that $p \in U$ there exists an open subset $W$ of $T_{4}$ such that $p \in W$ and $W \subseteq U$ and $N_{1}$ misses $\operatorname{Fr} W$. 
(39) Let $N_{1}$ be a subset of $T_{4}$. Suppose $T_{4} \uparrow N_{1}$ is second-countable. Then $N_{1}$ is finite-ind and ind $N_{1} \leq 0$ if and only if there exists a basis $B$ of $T_{4}$ such that for every subset $A$ of $T_{4}$ such that $A \in B$ holds $N_{1}$ misses $\operatorname{Fr} A$.

(40) Let $N_{1}, A$ be subsets of $T_{4}$. Suppose $T_{4}\left\lceil N_{1}\right.$ is second-countable and $N_{1}$ is finite-ind and $A$ is finite-ind and ind $N_{1} \leq 0$. Then $A \cup N_{1}$ is finite-ind and $\operatorname{ind}\left(A \cup N_{1}\right) \leq$ ind $A+1$.

\section{REFERENCES}

[1] Grzegorz Bancerek. The fundamental properties of natural numbers. Formalized Mathematics, 1(1):41-46, 1990.

[2] Grzegorz Bancerek. The ordinal numbers. Formalized Mathematics, 1(1):91-96, 1990.

[3] Leszek Borys. Paracompact and metrizable spaces. Formalized Mathematics, 2(4):481485, 1991.

[4] Czesław Byliński. Some basic properties of sets. Formalized Mathematics, 1(1):47-53, 1990.

[5] Agata Darmochwał. Compact spaces. Formalized Mathematics, 1(2):383-386, 1990.

[6] Agata Darmochwał. Finite sets. Formalized Mathematics, 1(1):165-167, 1990.

[7] Roman Duda. Wprowadzenie do topologii. PWN, 1986.

[8] Adam Grabowski. Properties of the product of compact topological spaces. Formalized Mathematics, 8(1):55-59, 1999.

[9] Krzysztof Hryniewiecki. Basic properties of real numbers. Formalized Mathematics, 1(1):35-40, 1990

[10] Robert Milewski. Bases of continuous lattices. Formalized Mathematics, 7(2):285-294, 1998.

[11] Andrzej Nędzusiak. $\sigma$-fields and probability. Formalized Mathematics, 1(2):401-407, 1990.

[12] Beata Padlewska. Families of sets. Formalized Mathematics, 1(1):147-152, 1990.

[13] Beata Padlewska and Agata Darmochwał. Topological spaces and continuous functions. Formalized Mathematics, 1(1):223-230, 1990.

[14] Karol Pąk. Basic properties of metrizable topological spaces. Formalized Mathematics, 17(3):201-205, 2009, doi: 10.2478/v10037-009-0024-8.

[15] Alexander Yu. Shibakov and Andrzej Trybulec. The Cantor set. Formalized Mathematics, 5(2):233-236, 1996.

[16] Andrzej Trybulec. Domains and their Cartesian products. Formalized Mathematics, 1(1):115-122, 1990.

[17] Andrzej Trybulec. A Borsuk theorem on homotopy types. Formalized Mathematics, 2(4):535-545, 1991.

[18] Michał J. Trybulec. Integers. Formalized Mathematics, 1(3):501-505, 1990.

[19] Zinaida Trybulec. Properties of subsets. Formalized Mathematics, 1(1):67-71, 1990.

[20] Mirosław Wysocki and Agata Darmochwał. Subsets of topological spaces. Formalized Mathematics, 1(1):231-237, 1990.

Received June 29, 2009 